\title{
Self-Heterodyne OFDM Transmission for Frequency Selective Channels
}

\author{
Nirmal Fernando, Student Member, IEEE, Yi Hong, Senior Member, IEEE, and Emanuele Viterbo, Fellow, IEEE
}

\begin{abstract}
Self-heterodyne OFDM (self-het OFDM) is known to provide complete immunity against frequency-offset and phase noise, with a much lower RF frontend complexity, when compared to conventional OFDM techniques. Self-het OFDM is considered to be a promising physical layer technology for millimeter-wave RF communications, where the implementation of low complexity stable oscillators is technically difficult. Although self-het OFDM has great potential, it has only been studied for additive white Gaussian noise and two-ray channel models. In this paper, we analyze the performance of self-het OFDM for general frequency selective channels and show that the standard self-het OFDM undergoes an outage if the RF carrier is affected by deep fading. In order to avoid this, we introduce a new technique called smart carrier positioning. We show both analytically and by simulation that the smart carrier positioning can improve the diversity order and the performance of standard self-het OFDM by approximately $4 \mathrm{~dB}$ at bit error rate of $10^{-2}$. In addition, we investigate the optimum power allocation between the carrier and the OFDM subcarriers under frequency selective conditions.
\end{abstract}

Index Terms-Non-coherent, OFDM, peak detection, selfheterodyne, DDO-OFDM, millimeter-wave communication, super-heterodyne, phase noise, low complexity receivers.

\section{INTRODUCTION}

$\mathbf{O}$ Rthogonal frequency division multiplexing (OFDM) is widely used in modern wireless communication systems due to its high spectral efficiency, good immunity against intersymbol-interference (ISI), simple channel equalization and simple integration to other physical layer technologies, such as multiple-input multiple-output (MIMO). However, OFDM is very sensitive to Doppler shift and oscillator instabilities, i.e., frequency-offset and phase noise [1]. For example, in millimeter-wave RF technologies, the implementation of stable oscillators is technically difficult and advanced frequency stabilization techniques are required [2], [3]. This results in a complex transceiver structure with high implementation cost. Hence, new OFDM techniques that are robust to oscillator instabilities and equipped with low complexity transceiver structures may have great potential in millimeter-wave RF communication.

One such OFDM technique, known as Flip-OFDM, encodes information only into the amplitude or the intensity of the carrier signal [4], [5]. Hence, Flip-OFDM is not affected by

Manuscript received July 15, 2012; revised October 14 and December 20, 2012. The associate editor coordinating the review of this paper and approving it for publication was H. Arslan.

The authors are with the Department of Electrical and Computer System Engineering, Monash University, Clayton, VIC 3800, Australia (e-mail: \{Nirmal.Fernando, Yi.Hong, Emanuele.Viterbo\} @monash.edu).

Digital Object Identifier 10.1109/TCOMM.2013.021913.120510 phase noise. Although it has excellent power efficiency characteristics, it is spectrally inefficient compared to conventional OFDM schemes.

Taking a different approach, Shoji et al. proposed the selfheterodyne down-conversion technique to compensate for high level oscillator instabilities in $60 \mathrm{GHz} \mathrm{RF}$ communications using QAM/OFDM signalling [6], [7]. They experimentally demonstrated that the performance of the self-heterodyne OFDM receiver is not affected by RF phase noise [6], [8], [9]. In this paper, we denote this technique as self-het OFDM. A similar concept was independently proposed for optical communications, where the OFDM technique, named Direct-Detection Optical OFDM (DDO-OFDM), was used to compensate for chromatic dispersion in long-haul optical transmissions [10], [11].

In [6], [7], Shoji et al. showed that self-heterodyne is a major deviation from conventional super-heterodyne RF structures, where the transmitter sends both local carrier and modulated signals. At the receiver, a square-law device or circuitry (i.e. self-mixing) is used to down-convert the RF signal, without the need of a local carrier, carrier phase recovery and carrier frequency correction. Hence, the complexity of RF front end in self-het OFDM receiver is significantly lower than that of conventional OFDM schemes with super-heterodyne RF structures. In addition, since the transmitter can ensure that the local carrier phase is synchronized with the OFDM subcarriers, the self-het OFDM down-conversion is extremely stable and theoretically has zero phase noise. However, self-het OFDM has the following three disadvantages: (i) it generates intermodulation distortions during the self-mixing process; (ii) it uses only up to $50 \%$ of the available spectrum for communications; and (iii) approximately half of the transmitter power is used for the local carrier transmission. As a remark, PAPR of self-het OFDM is similar to that of conventional OFDM schemes with the same number of information subcarriers. Since OFDM schemes have high PAPR ratios, the nonlinear distortion can degrade the performance to some extent, but it is common for both schemes.

After the introduction of self-het OFDM techniques, there has been a number of recent developments. In [8], the bit error rate (BER) performance of self-het OFDM over additive white Gaussian noise (AWGN) channels was analyzed. In [12], antenna arrays at the receiver were employed in self-het OFDM to increase the sensitivity for two-ray channels, providing an SNR improvement similar to maximal-ratio combining schemes. In [13], an experimental demonstration of a singleinput multiple-output (SIMO) case was analyzed for two-path channel environments. In [14], the intermodulation distortion 
due to nonlinear detection (i.e., nonlinear square-law device) was investigated, when the millimeter-wave self-het OFDM was applied to multichannel analog signal transmissions. In [15], self-het OFDM was applied to wireless personal area network for $60 \mathrm{GHz}$ RF communications. In [16], adaptive modulation and coding was presented to exploit the frequency selective nature in the $60 \mathrm{GHz} \mathrm{RF}$ band for wireless indoor networks.

Although self-het OFDM is mainly studied for $60 \mathrm{GHz}$ RF band, it can be potentially applicable to other millimeterwave RF frequency bands such as E-band $(60 \mathrm{GHz}-90 \mathrm{GHz})$ and unlicensed terahertz RF bands [17]. In those frequency ranges, the implementation of RF front-end components to conventional OFDM (with super-heterodyne structures) is extremely difficult and expensive. To avoid this, one approach is to use self-het OFDM that can trade some RF spectrum for lower complexity receiver. In particular, self-het OFDM has a simple and stable RF front-end, since the receiver does not require: $(i)$ carrier phase recovery circuit (i.e. PLL), (ii) stable oscillators and (iii) complex frequency stabilization techniques. Hence, self-het OFDM is a good solution to implement low complexity short range mobile communication systems, delivering high speed data within such RF bands.

In the research literature, self-het OFDM has only been studied for AWGN and two ray channels, and the performance of self-het OFDM has never been analyzed for frequency selective channels to the best of our knowledge. In this paper, we focus on self-het OFDM for general frequency selective channels, that appear in certain cases in millimeter-wave RF channels [18]. The main contributions of our paper are:

- A comprehensive analysis of self-het OFDM over frequency selective channels: we first consider the equivalent baseband model and the appropriate channel equalization techniques and then we analyze the BER performance.

- Enhancements for self-het OFDM: we show that the standard self-het OFDM reception experiences an outage if the transmitted local carrier is affected by deep fading. As an enhancement, we present a simple technique, named smart carrier positioning, to significantly reduce the occurrence of the outage and hence improve the BER performance. We verify such performance analytically and by computer simulations. Furthermore, we discuss how to allocate transmission powers between the local carrier and OFDM subcarriers in order to minimize the error performance of the system.

In this paper, we do not consider the link budget issues, since we compare the systems performance based on the receiver SNRs. Therefore, we will not discuss the transmitter power, the transmission range, the transceiver antenna directivity issues.

The paper is organized as follows. In Section II, we discuss the frequency selectivity in millimeter wave RF bands. Subsequently, we review self-het OFDM communications over AWGN channels. In Section III, we provide a comprehensive analysis of self-het OFDM over frequency selective channels and compare it with that over AWGN channels. In Section IV, we present the enhanced self-het OFDM schemes. In
Section V, we show the simulation results. Finally, we draw the conclusions in Section VI.

Throughout this paper, we adopt the following notation: $\Re\{\cdot\}$ denotes the real component of a complex number, $j$ denotes the imaginary unit, $*$ represents the convolution operation, $\delta(\cdot)$ denotes dirac delta function, $|a|$ represents the absolute value of $a, G_{x}$ represents the power spectral density (PSD) of signal $x(t), f_{X}(x)$ denotes the probability distribution function of random variable $X$, and $Q(x)=$ $\frac{1}{2 \pi} \int_{x}^{\infty} \exp \left(-u^{2} / 2\right) d u$.

\section{System MOdeL}

\section{A. Frequency selectivity of millimeter wave RF channels}

In $60 \mathrm{GHz}$ RF bands, the small-scale fading due to the multipath propagation is studied in [19] and references therein. It is noted that the channel impulse response of $60 \mathrm{GHz}$ radio link is typically ray like, and both modified SV models [20], [21], [22] and tapped delay line models [23] are mainly used to characterize these channels.

In [18], the frequency selectivity of $60 \mathrm{GHz}$ RF channels is analyzed experimentally for both line-of-sight (LoS) and non-LoS (NLoS) in indoor radio environments. The channel coherence bandwidth, denoted by $B c_{0.9}$ [18], a measurement that is inversely proportional to the degree of the channel frequency selectivity, is highly related to the root-mean-squared delay spread of channel impulse response $\sigma_{\tau}$. For $60 \mathrm{GHz}$ NLoS channels, it is observed that the coherence bandwidth $B c_{0.9}$ can vary from $2 \mathrm{MHz}$ to $20.52 \mathrm{MHz}$. For both LoS and NLoS cases, when the transceivers have omni directional antennas, $B c_{0.9}$ is comparatively small (3-15.4 MHz for LoS and 2.6-6.5 MHz for NLoS). For LoS case, with misaligned directional antennas, channel coherent bandwidth $B c_{0.9}$ can vary from 3.2 MHz to $44.7 \mathrm{MHz}$. Hence, frequency selectivity can occur in the above cases whenever the signal bandwidth is greater than the above $B c_{0.9}$.

\section{B. Self-Het OFDM over AWGN channels}

A block diagram of a typical self-het OFDM communications system is shown in Fig. 1 , where $N, N_{x}$, and $N_{g}$ represent the size of Inverse Fast Fourier Transform (IFFT), the number of OFDM subcarriers that are used to encode information, and the number of subcarriers omitted, respectively. We note that $N=N_{x}+N_{g}$.

At the transmitter, the information is first mapped to OFDM symbols $X_{k}, k=1,2, \ldots, N_{x}$, using $M$-QAM signaling and then sent over $N_{x}$ OFDM subcarriers ${ }^{1}$. The time-domain OFDM symbol is generated by adding the cyclic prefix (CP) after the IFFT operation, which has a length sufficient to avoid inter-symbol-interference (ISI). Then, both parallel-to-serial conversion and digital-to-analogue conversion are performed at the transmitter to generate the OFDM signal $s(t)$. Let $f_{c}$

\footnotetext{
${ }^{1}$ Note that a practical implementation of the transmitter can use an $N_{x}$ point IFFT followed by a frequency shift. We adopted the model in Fig. 1 for the better visualization of the standard self-het OFDM. However, it is useful to add the local RF carrier externally, since it saves $50 \%$ in complexity of the FFT/IFFT.
} 


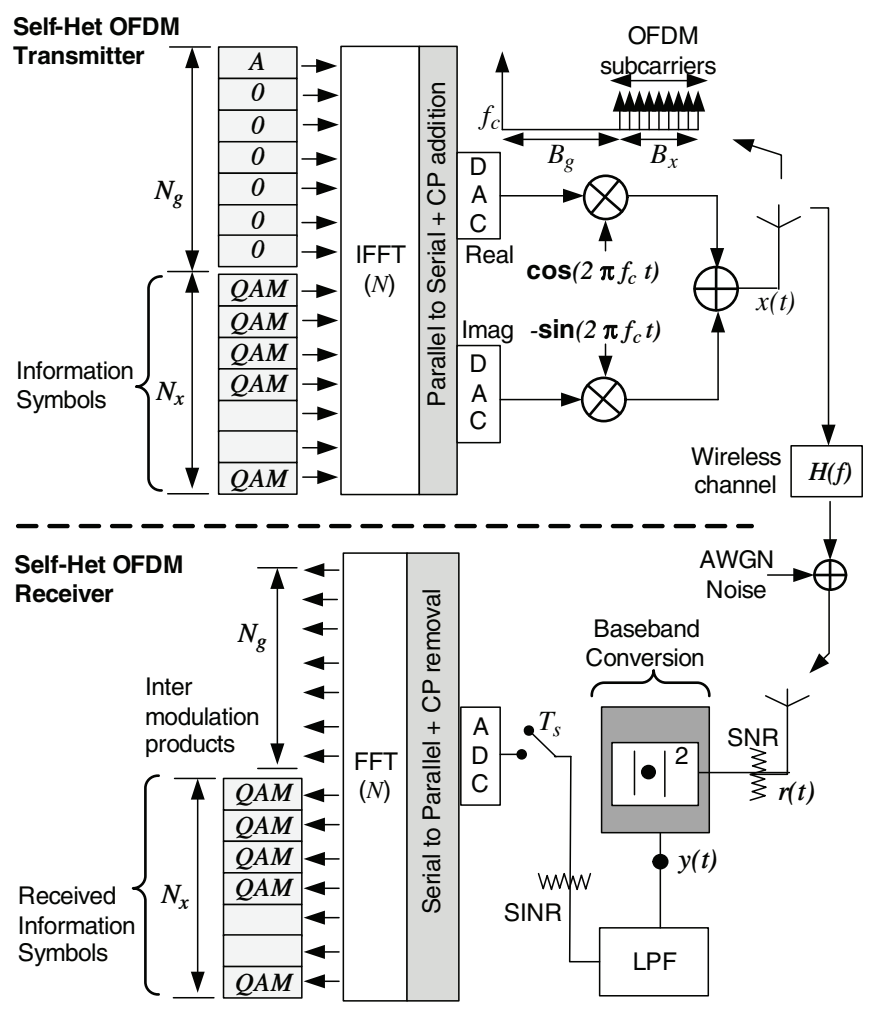

Fig. 1. Self-Het OFDM communication system.

and $\Delta f$ denote RF carrier frequency and the OFDM carrier spacing, respectively. Then the OFDM signal is given by

$$
s(t)=\Re\left\{\sum_{k=1}^{N_{x}} X_{k} e^{j 2 \pi\left(f_{c}+B_{g}+k \Delta f\right) t}\right\}
$$

where $B_{g}=\Delta f N_{g}$. The RF signal $x(t)$ can be generated by adding a carrier signal to $s(t)$ with a frequency gap of $B_{g}$ between the carrier and the first OFDM subcarrier, i.e.,

$$
x(t)=A \cos \left(2 \pi f_{c} t\right)+s(t) .
$$

If $x(t)$ is transmitted over an AWGN channel, the received electrical signal after the receiver antenna is given by

$$
r(t)=x(t)+n(t)
$$

where $n(t) \sim \mathcal{N}\left(0, \sigma^{2}\right)$ is AWGN with noise power $\sigma^{2}$.

At the receiver, the nonlinear operation is performed as

$$
y(t)=|r(t)|^{2} .
$$

Since the carrier signal is embedded in the received signal, the operation in (4) directly converts the passband signal to the baseband signal without the needs of local carrier generation, carrier phase recovery, carrier frequency correction and complex down-conversion circuitries. However, the operation in (4) creates a number of inter-modulation products due to selfand inter-mixing of individual signal component in $r(t)$. In order to understand the composition of $y(t)$, we will analyze the power spectral density (PSD) of $y(t)$, denoted by $G_{y}(f)$,
[24], [25],

$$
\begin{aligned}
G_{y}(f) & =\underbrace{2 G_{s}(f) * \frac{A^{2}}{4}\left\{\delta\left(f-f_{c}\right)+\delta\left(f+f_{c}\right)\right\}}_{\{1\}} \\
& +\underbrace{G_{s}(f) * G_{s}(f)}_{\{2\}}+\underbrace{G_{n}(f) * G_{n}(f)}_{\{3\}} \\
& +\underbrace{2 G_{n}(f) * \frac{A^{2}}{4}\left\{\delta\left(f-f_{c}\right)+\delta\left(f+f_{c}\right)\right\}}_{\{4\}} \\
& +\underbrace{2 G_{s}(f) * G_{n}(f)}_{\{5\}}+\left[\mathrm{DC}, 2 f_{c}\right. \text { components] (5) }
\end{aligned}
$$

where $G_{s}(f)$ and $G_{n}(f)$ are PSDs of $s(t)$ and $n(t)$, respectively. The expression in (5) is valid under the assumptions that:

- both $s(t)$ and $n(t)$ are zero-mean Gaussian random processes with PSD of $S_{0}$ and $\frac{N_{0}}{2}$;

- the numbers of subcarriers (i.e. $N$ and $N_{x}$ ) are sufficiently large.

The term $\{1\}$, generated by mixing of the carrier with the OFDM subcarriers, contains the power spectrum of the useful OFDM signal. The term $\{2\}$ is the auto-correlation of $G_{s}(f)$, accounting for unwanted tones. The term $\{3\}$ is generated through self-mixing of noise. The term $\{4\}$ is the noise that has been down-converted by mixing with the RF carrier. The term $\{5\}$ comes from mixing of noise with the OFDM subcarriers. We note that in order to separate the useful term $\{1\}$ from $\{2\}$ the guard band $B_{g}=\Delta f N_{g}$ must satisfy the condition

$$
B_{g} \geq B_{x} .
$$

where $B_{x}=\Delta f N_{x}$ represents the bandwidth of $s(t)$. Hence, the information contained in $\{1\}$ can be easily recovered at the receiver. Note that the sum of the terms $\{3\},\{4\}$ and $\{5\}$ account for the noise and interference impairments.

At the receiver, a low pass filter (LPF) with a cutoff frequency $B_{g}+B_{x}+B_{h}$ and a DC component filter are used where $B_{h}$ represents the upper guard band (see Fig. 2(a)). This will remove the high frequency and DC components before the sampling and the analog-to-digital conversion (ADC). Then, the $\mathrm{CP}$ is removed from the OFDM symbol and the FFT operation is performed after the serial-to-parallel conversion. As shown in Fig. 1, we observe that the first $N_{g}$ OFDM subcarriers are ignored and the next $N_{x}$ OFDM subcarriers are used to transmit the information symbols corresponding to $X_{k}$. Let $Y_{k}$ be the received information symbol of the $k$-th OFDM subcarrier, given by

$$
Y_{k}=A X_{k}+Z_{k}
$$

where $Z_{k}$ denotes the equivalent noise from the terms $\{3\}$, $\{4\}$ and $\{5\}$ in (5). We note that $Z_{k}$ is correlated with the input $X_{k}$, and the variance of $Z_{k}$ varies with $k$, where $k=$ $N_{g}+1, \ldots, N_{g}+N_{x}$. However, for large $N$, we can ignore the correlation of $Z_{k}$, since the FFT operation mixes the correlated noise to a level, where $Z_{k}$ can be assumed independent from the input $X_{k}$. 


\section{Performance analysis}

\section{A. Self-het OFDM over frequency selective channels}

In this section, we consider self-het OFDM over frequency selective channels. Let $h(t)$ be the frequency selective channel impulse response. Using the system model in Fig. 1, the received signal $r(t)$ is given by

$$
r(t)=h(t) * x(t)+n(t)
$$

where $x(t)$ is given in (2). The PSD of $r(t)$, denoted by $G_{r}(f)$, is now given by

$$
G_{r}(f)=\Lambda\left(f_{c}\right)+|H(f)|^{2} G_{s}(f)+G_{n}(f)
$$

where

$$
\Lambda_{c}(f) \triangleq \frac{A^{2}\left|H\left(f_{c}\right)\right|^{2}}{4}\left(\delta\left(f-f_{c}\right)+\delta\left(f+f_{c}\right)\right)
$$

and $H\left(f_{c}\right)$ denotes the channel response at carrier frequency. Fig. 2(a) shows $G_{r}(f)$ over frequency selective channels, where $G_{x}(f), H(f), S_{0}, B_{h}, B_{l}$ and $B_{m}$ represent the PSD of the transmitted signal, the channel response at the frequency $f$, the average power spectral density of $s(t)$, the guard band of higher frequency side, the guard band of lower frequency side and the total noise bandwidth, respectively.

Similar to the AWGN case, at the receiver, self-mixing in (4) is performed immediately after filtering the out-of-band noise power (see Fig. 1) and then converts the passband signal to the baseband signal. However, the baseband components generated are different from those in (5). The PSD of $y(t)$ is given by

$$
\begin{aligned}
G_{y}(f) & =G_{r}(f) * G_{r}(f) \\
& =\underbrace{2 \Lambda_{c}(f) *\left\{|H(f)|^{2} G_{s}(f)\right\}}_{\{1\}} \\
& +\underbrace{\left\{|H(f)|^{2} G_{s}(f)\right\} *\left\{|H(f)|^{2} G_{s}(f)\right\}}_{\{2\}} \\
& +\underbrace{G_{n}(f) * G_{n}(f)}_{\{3\}}+\underbrace{2 \Lambda_{c}(f) * G_{n}(f)}_{\{4\}} \\
& +\underbrace{2\left\{|H(f)|^{2} G_{s}(f)\right\} * G_{n}(f)}_{\{5\}} \\
& +\left[\text { DC, } 2 f_{c} \text { components }\right] .
\end{aligned}
$$

The individual terms in (10), as shown in Fig. 2(b), correspond to those defined in (5). As discussed in the AWGN case, the term $\{1\}$ of (10) contains the power spectrum of the useful OFDM signal, while the terms of $\{2\},\{3\},\{4\},\{5\}$ are the noise and interference impairments. It can be observed that the term $\{1\}$ depends on $H\left(f_{c}\right)$ due to the term $\Lambda_{c}(f)$. It must be noted that some of the resulting baseband components are generated from the negative frequency parts of the underlying double-sided PSDs. However, if the condition in (6) applies, there is no interference between terms $\{1\}$ and $\{2\}$, and hence, the information contained in $\{1\}$ can be filtered easily at the receiver.

Unlike in AWGN channel, the received carrier signal is faded independently, and this faded signal is used for the down-conversion. Hence, the received information symbols

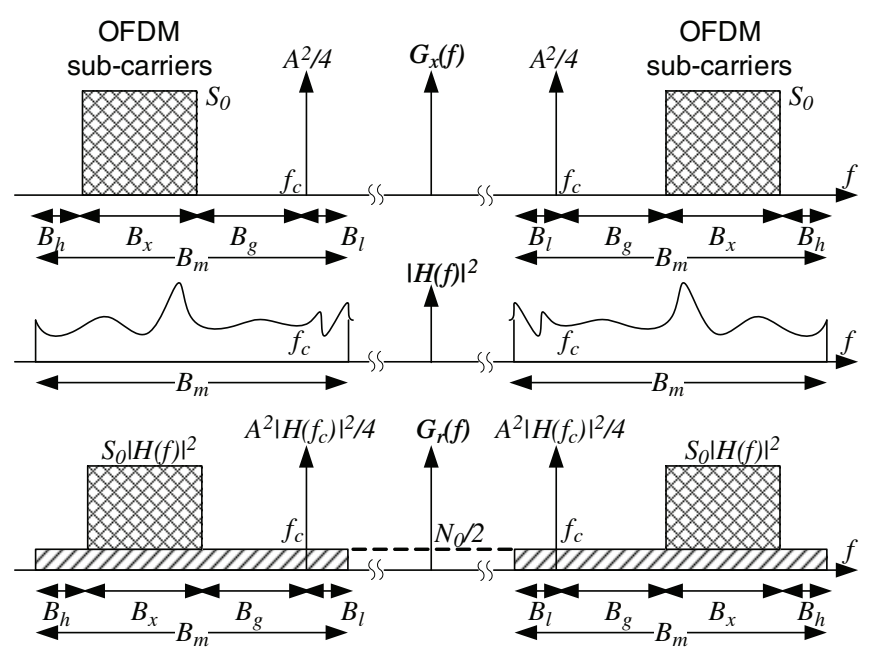

(a)
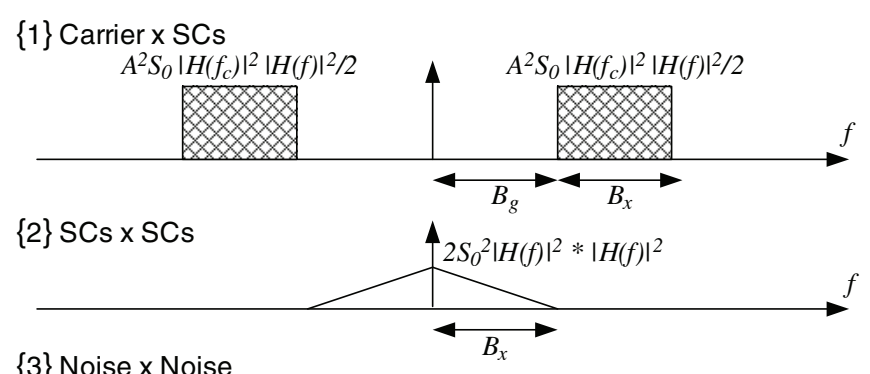

$\{3\}$ Noise $x$ Noise

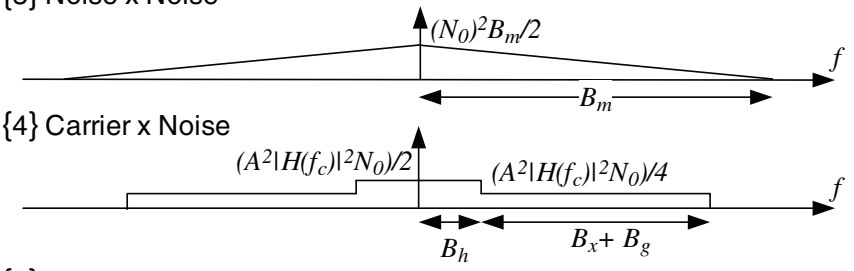

$\{5\}$ SCs $x$ Noise

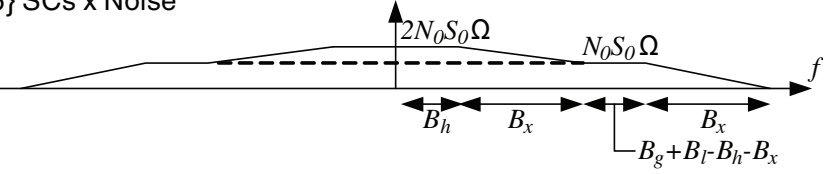

(b)

Fig. 2. (a) PSD of the transmitted signal and the received signal; (b) baseband components after the down-conversion with absolute square operation. $\Omega=$ $\int_{f_{c}+B_{g}}^{f_{c}+B_{g}+B_{x}}|H(f)|^{2} d f$.

depends on the channel response at the carrier frequency, and the equivalent discrete baseband model can be written as

$$
Y_{k}=A H_{c}{ }^{*} H_{k} X_{k}+Z_{k}
$$

where $H_{c}, H_{k}, Y_{k}$ and $Z_{k}$ are the equivalent channel response at the carrier, the equivalent channel response at $k$-th OFDM subcarrier, the received information symbol in the $k$-th OFDM subcarrier and the equivalent noise component contributed by the terms in $\{3\},\{4\},\{5\}$ in (10), respectively. Although self-het OFDM receiver does not recover the phase of the carrier, the equivalent baseband channel coefficient $H_{k}$ can be estimated at the receiver if a known transmitter symbol pattern is transmitted (i.e. OFDM training sequence). Hence, the conventional channel equalization techniques can be used at the receiver to compensate any frequency selective channel effects. 


\section{B. Performance Analysis}

In this section, we will analyze the BER performance of self-het OFDM over frequency selective channels. For the sake of simplicity, we assume $B_{l}=B_{h}=0$. In Fig. 2(b), the signal-to-interference-noise ratio (SINR) of the $k$-th OFDM subcarrier $\gamma_{k}$ can be obtained as

$$
\gamma_{k}=\frac{\frac{A^{2}}{2}\left|H_{c}\right|^{2}\left|H_{k}\right|^{2} S_{0}}{\frac{A^{2}\left|H_{c}\right|^{2} N_{0}}{4}+\frac{N_{0}^{2} B_{m}\left(N_{g}+N_{x}-k\right)}{2\left(N_{g}+N_{x}\right)}+S_{0} N_{0} \Delta f \lambda(k)}
$$

where

$$
\lambda(k) \triangleq \frac{2\left(N_{g}+N_{x}-k\right)}{N_{g}+N_{x}} \sum_{k=N_{g}+1}^{N_{g}+N_{x}}\left|H_{k}\right|^{2} .
$$

Furthermore, we define the power ratio between the RF carrier and the OFDM subcarriers as

$$
\eta \triangleq \frac{\frac{1}{2} A^{2}}{2 S_{0} B_{x}} .
$$

According to (12), the SINR of the $k$-th OFDM subcarrier depends on the $\left|H_{c}\right|^{2}$ and $\left|H_{k}\right|^{2}$ distributions. To simplify notation, we define

$$
\xi \triangleq\left|H_{c}\right|^{2} \text { and } \zeta_{k} \triangleq\left|H_{k}\right|^{2} .
$$

In general, $\xi$ and $\zeta_{k}$ undergo independent fading since the carrier and OFDM subcarries are separated by a considerable band gap $\left(B_{g}\right)$. If we assume that the channel is Rayleigh fading (i.e. $H_{c}, H_{k} \sim \mathcal{N}_{C}(0,1)$ ), then both $\xi$ and $\zeta_{k}$ have exponential distributions. Substituting (14) into (12) gives

$$
\gamma_{k}=\frac{\eta \xi \zeta_{k} \bar{\gamma}}{\eta \xi+\left(\frac{1}{\bar{\gamma}}\right)\left(\frac{N_{g}+N_{x}-k}{N_{x}}\right)+\frac{\lambda(k)}{N_{x}}}
$$

where $\bar{\gamma} \triangleq \frac{2 S_{0}}{N_{0}}$. In the SNR region of interest (e.g., $\bar{\gamma}>10$ $\mathrm{dB}$ ), the second term of the denominator in (16) is comparatively insignificant, when compared to the first and third terms. For an example, considering a frequency flat channel (i.e. $\zeta_{k}=\xi=1$ for all $k$ ) and $B_{g}=B_{x}$ (i.e., $N_{g}=N_{x}$ ), then (16) further simplifies to

$$
\begin{aligned}
\gamma_{k} & =\frac{\eta \bar{\gamma}}{\eta+\frac{\left(2 N_{x}-k\right)}{N_{x}}\left[\frac{1}{\bar{\gamma}}+1\right]} \\
& \approx \frac{\eta \bar{\gamma}}{\eta+\frac{\left(2 N_{x}-k\right)}{N_{x}}}
\end{aligned}
$$

for $\bar{\gamma}>>1$. Hence, (16) can be rewritten as

$$
\gamma_{k} \approx \frac{\eta N_{x} \xi \zeta_{k} \bar{\gamma}}{\eta N_{x} \xi+\lambda(k)}
$$

If $M$-QAM information symbols are used to transmit the data, (as proved in Appendix A), the average BER of $k$-th OFDM subcarrier can be given as

$$
\begin{aligned}
\mathrm{BER}_{k}= & \frac{2}{\log _{2}(M)}\left(1-\frac{1}{\sqrt{M}}\right)\left(1-\frac{1}{2} \sqrt{\frac{3 \pi \bar{\gamma}}{3 \bar{\gamma}+2(M-1)}}\right. \\
& \left.\times U\left[\frac{1}{2}, 0, \frac{2(M-1) \lambda(k)}{\eta N_{x}\{3 \bar{\gamma}+2(M-1)\}}\right]\right)
\end{aligned}
$$

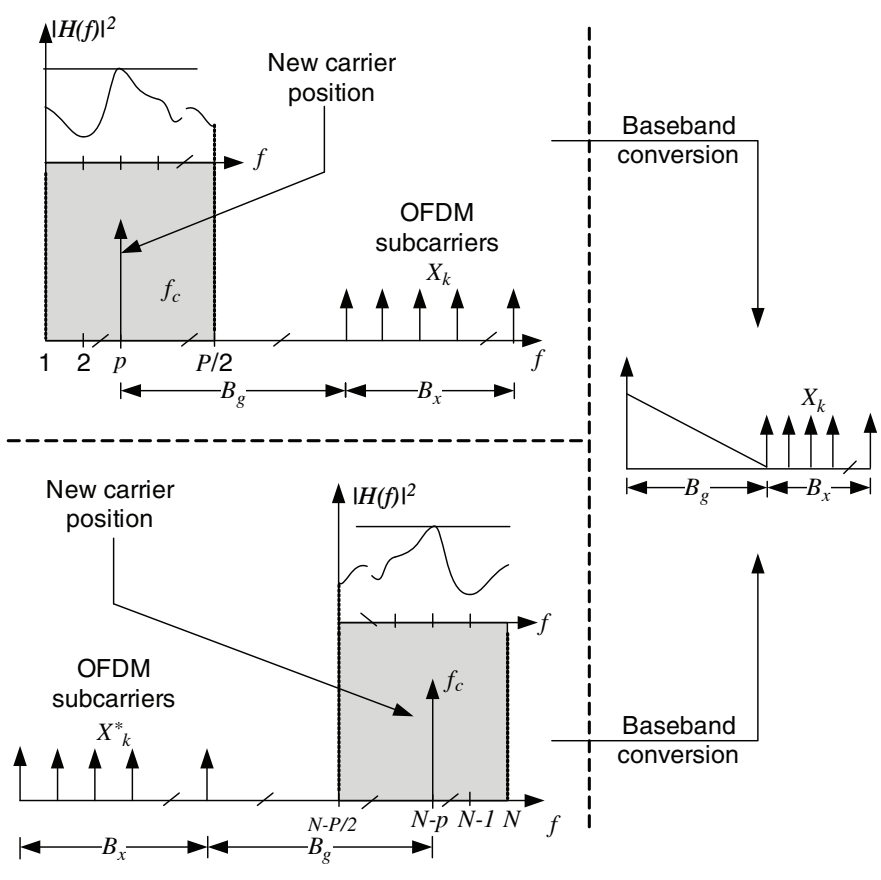

Fig. 3. Smart carrier positioning.

where $U(a, b, z)$ is defined in (31). The overall BER of the system is the average $\mathrm{BER}_{k}$ across the OFDM subcarriers used to transmit information symbols, i.e.,

$$
\mathrm{BER} \triangleq \frac{1}{N_{x}} \sum_{k=N_{g}+1}^{N_{g}+N_{x}} \mathrm{BER}_{k}
$$

and we note that BER is a function of $\eta$.

\section{EnhancEMEnTs FOR SElF-Het OFDM}

\section{A. Smart Carrier Positioning}

The standard self-het OFDM signal undergoes an outage if the local carrier experiences deep fading. This can be seen in the equivalent baseband model given in (11), where $Y_{k}$ depends on $H_{c}$. To reduce the probability of the outage, we introduce the smart carrier positioning technique for pointto-point wireless communication. In this technique, the carrier can be positioned either on the left or the right handside of the OFDM subcarriers, as shown in Fig. 3. In the latter case, the information subcarriers are transmitted in the lower band. In the smart carrier positioning technique, the carrier can be placed in the $\hat{i}$-th position such that,

$$
\begin{aligned}
\hat{i}= & \underset{i \in\left\{1, \ldots \frac{P}{2},\right.}{\arg \max }\left\{\left|H_{i}\right|^{2}\right\} \\
& \left.N-\frac{P}{2}, \ldots, N\right\}
\end{aligned}
$$

where $\left|H_{\hat{i}}\right|^{2}$ is the channel gain at the carrier. $P$ denotes the total number of potential carrier positions. Let $p=\hat{i}$ if the carrier is positioned on left handside, or let $p=N+1-\hat{i}$ if the right handside. Then $B_{g}=B_{x}=\Delta f(N+1-p) / 2$ where $p \in 1,2,3, \ldots, P / 2$. We note that if the carrier is placed on the right handside, as shown in Fig. 3, the transmitted OFDM symbols must be $X_{k}{ }^{*}$, instead of $X_{k}$, in order to receive the information symbols correctly. 
Note that smart carrier positioning technique requires very limited feedback information at the transmitter. Specifically, it needs to know at which frequency the carrier must be positioned. The receiver can estimate the optimum RF carrier location from the available channel state information, and send it back to the transmitter. We note this feedback message is very short (i.e., it requires a limited number of information bits to encode the index $\hat{i}$ ).

Let us define $\xi \triangleq\left|H_{\hat{i}}\right|^{2}$. If we assume that the channel is Rayleigh fading, and all $P$ carrier positions undergo independent fading, the cdf of $\xi$ is given as

$$
\begin{aligned}
F_{\xi}(z) & =\prod_{k=1}^{P / 2} F_{\zeta_{k}}(z) \times \prod_{k=N-P / 2}^{N} F_{\zeta_{k}}(z) \\
& =(1-\exp (-z))^{P}
\end{aligned}
$$

where $F_{\zeta_{k}}(z)$ is the cdf of the channel gain of $k$-th OFDM subcarrier. Then the pdf of $\xi$ is given by,

$$
f_{\xi}(z)=P \exp (-z)(1-\exp (-z))^{P-1} .
$$

For $M$-QAM signalling, as shown in Appendix A, the average BER of the $k$-th OFDM subcarrier using the smart carrier positioning technique can be proved as

$$
\begin{array}{r}
\operatorname{BER}_{k}^{S}=\frac{2}{\log _{2}(M)}\left(1-\frac{1}{\sqrt{M}}\right)\left(1-\frac{1}{2} \sqrt{\frac{3 \pi \bar{\gamma}}{3 \bar{\gamma}+2(M-1)}}\right. \\
\left.\sum_{t=1}^{P}\left(\begin{array}{c}
P \\
t
\end{array}\right)(-1)^{t-1} U\left[\frac{1}{2}, 0, \frac{2(M-1) \lambda(k) t}{\eta N_{x}\{3 \bar{\gamma}+2(M-1)\}}\right]\right)
\end{array}
$$

where $\lambda(k)$ and $U(a, b, z)$ are defined in (13) and (31), respectively. The overall BER, as given in (18), can be obtained by averaging $\mathrm{BER}_{k}^{S}$ over all subcarriers,

$$
\mathrm{BER}^{S} \triangleq \frac{1}{N_{x}} \sum_{k=N_{g}+1}^{N_{g}+N_{x}} \operatorname{BER}_{k}^{S}
$$

We notice that $\mathrm{BER}^{S}$ depends on $\bar{\gamma}\left(\right.$ i.e. $\left.\frac{2 S_{0}}{N_{0}}\right), \eta$, and $P$.

\section{B. Power allocation between the carrier and OFDM subcar- riers}

In self-het OFDM, the performance of the communication system depends on the power allocation $\eta$ in (14) between the carrier and the OFDM subcarrier. This is evident from (18) and (22), where the overall BER performance depends on $\eta$. Let $P_{t}$ be the overall transmit power in a self-het OFDM transmitter. For the standard self-het OFDM, the optimum $\eta$ can be found by

$$
\eta_{\mathrm{opt}}=\underset{\eta}{\arg \min }\{\mathrm{BER}\}
$$

subject to

$$
P_{t}=\frac{A^{2}}{2}+2 B_{x} S_{0}
$$

where BER is given in (18). For enhanced self-het OFDM, $\mathrm{BER}^{S}$ in (22) is used in (23) and $\eta_{\text {opt }}$ can be found accordingly.
From the definition of $\eta$ in (14) and (24), we have

$$
\begin{aligned}
\bar{\gamma} & =\frac{P_{t}}{B_{x} N_{0}(\eta+1)} \\
& =\frac{N_{x}+N_{g}}{N_{x}(\eta+1)} \gamma_{c}
\end{aligned}
$$

where $\gamma_{c}$ is the equivalent coherent SNR before the baseband conversion, defined as

$$
\gamma_{c} \triangleq \frac{P_{t}}{N_{0}\left(B_{g}+B_{x}\right)} .
$$

The optimum $\eta_{\text {opt }}$ can be selected by substituting $\bar{\gamma}$ given in (25) to (22) and solving

$$
\frac{\partial \mathrm{BER}}{\partial \eta}=0 \quad \text { and } \quad \frac{\partial \mathrm{BER}^{S}}{\partial \eta}=0
$$

for standard and enhanced self-het OFDM, respectively. For a given system with known $P, B_{g}, B_{x}, \frac{S_{0}}{N_{0}}$, we can numerically solve (27) and find $\eta_{\text {opt }}$ for both cases. Substituting corresponding $\eta_{\text {opt }}$ in (18) and (22) yields the optimum theoretical BER for standard and enhanced self-het OFDMs, which will be simulated in the following section.

\section{Simulation Results}

In this section, we analyze the performance of self-het OFDM over multi-path fading channels by conducting the following simulations:

1) an illustration of PSDs of the useful and noise/interference signals generated during the self-het OFDM baseband conversion;

2) BER performance comparison of self-het-OFDM over AWGN and Rayleigh fading channels;

3) BER performance comparison of self-het-OFDM with and without smart carrier positioning technique; and

4) an illustration of optimum power allocation ratio $\eta_{o p t}$ under different $\gamma_{c}$, as well as BER performance comparison of self-het OFDM with and without optimum power allocation.

A summary of key simulation parameters is given in Table I. We assume that we have $B_{h}=B_{l}=0$ in all simulations, and the radio link operates under NLoS with omni directional antennas (i.e. Rayleigh fading). We note that the channel is subject to frequency selectivity, since the signal bandwidth $B_{x}+B_{g}$ (e.g. $100 \mathrm{MHz}$ ) is significantly greater than the channel coherent bandwidth $B c_{0.9}$ discussed in Section II(A).

For Rayleigh fading channels, Fig. 4 compares the analytical PSDs of signal components in (10) with the PSDs obtained by simulations at $\gamma_{c}=15 \mathrm{~dB}$. The simulated PSDs are obtained by averaging the signal powers generated in 10000 channel realizations. It is shown that they match each other and the useful signal component, term $\{1\}$ in (10), does not interfere with the term $\{2\}$ (i.e. Subcarriers $\times$ Subcarriers), since $N_{x}=N_{g}$. However, the terms $\{3\},\{4\}$ and $\{5\}$ in (10) act as noise/interference for useful OFDM subcarriers (e.g. from $k=257,258, \ldots, 512$ ). It can be seen that the majority of noise power is contributed by the terms $\{4\}$ (i.e. Noise $\times$ Carrier) and $\{5\}$ (i.e. Noise $\times$ Subcarrier) in (10). Hence, we can ignore the term $\{3\}$ (i.e. Noise $\times$ Noise) in the SNR region 
TABLE I

SIMULATION PARAMETERS

\begin{tabular}{l|c}
\hline Parameter & value \\
\hline \hline Carrier and subcarrier power ratio $(\eta)$ & 1 \\
Number of OFDM subcarrier $\left(N_{x}\right)$ & 256 \\
Bandgap between the carrier \& subcarriers $\left(N_{g}\right)$ & 256 \\
upper and lower bandgap of filter $\left(B_{l}, B_{g}\right)$ & 0 \\
IFFT/FFT size $(N)$ & 512 \\
Sampling time $\left(T_{s}\right)$ & $10 \mathrm{~ns}$ \\
Channel length & $32 T_{s}$ \\
Cyclic prefix $(\Delta)$ & $(32+1) T_{s}$ \\
OFDM symbol duration $\left(T_{s} N\right)$ & $5.12 \mu \mathrm{s}$ \\
Wireless fading channel model & Rayleigh, AWGN \\
Modulation & QPSK, 16-QAM \\
\hline
\end{tabular}

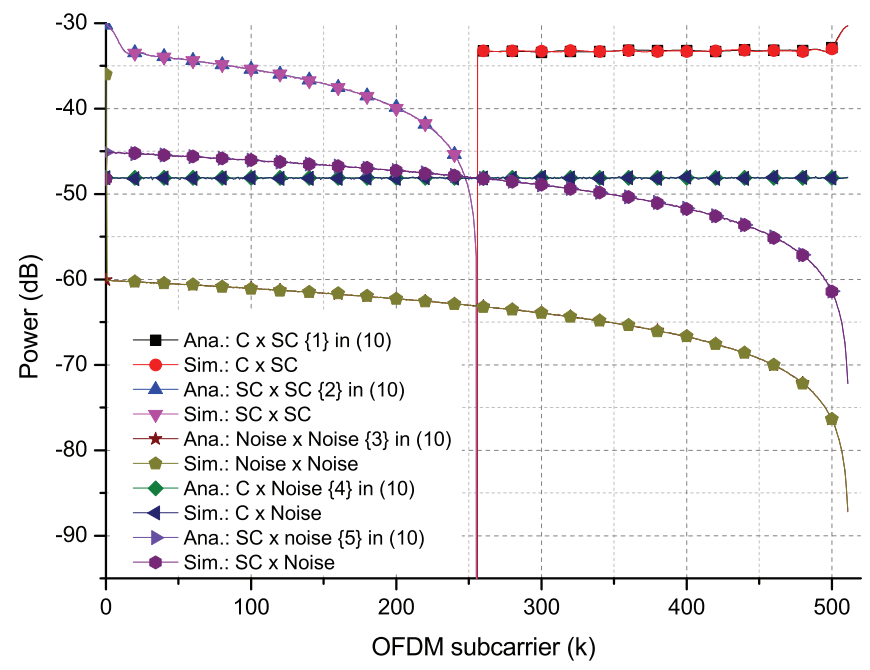

Fig. 4. Analytical and simulated PSDs of signal components generated in self-het OFDM baseband conversion.

of interest. Since the PSD of $\{5\}$ in (10) gradually decaying with frequency, the SINR after the baseband conversion $\left(\gamma_{k}\right)$ improves accordingly.

Fig. 5 compares the simulated SINRs at $\gamma_{c}=10,15$ and $20 \mathrm{dBs}$, respectively, with the analytical $\gamma_{k}$ in (17). We observe how they agree with the simulation results. We note that $\gamma_{k}$ increases as the subcarrier indices. The SINR gap between the first (i.e. $k=257$ ) and last OFDM subcarrier (i.e. $k=512$ ) is approximately $3.3 \mathrm{~dB}$. Hence, the BER performance of OFDM subcarriers with large subcarrier indices are better than those located closer to the RF carrier.

Fig. 6 compares the BER performance of standard self-het OFDM and conventional OFDM with super-heterodyne structures for AWGN and Rayleigh fading channels, respectively. In this simulation, we assume no phase noise generated at the receiver. With QPSK signalling, since half of OFDM subcarriers are omitted in self-het OFDM, the spectral efficiency of selfhet OFDM is $50 \%$ of that achieved by conventional OFDM scheme. Hence, we adopt 16-QAM and QPSK signallings for self-het OFDM and conventional OFDM, respectively, in order to have the same spectral efficiency. For AWGN channels, we observe, in Fig. 6, that the BER performance self-het OFDM is approximately $9 \mathrm{~dB}$ worse than that of the conventional OFDM scheme. For Rayleigh fading channels, we see that self-het OFDM (16-QAM) perform $10.5 \mathrm{~dB}$ worse than conventional

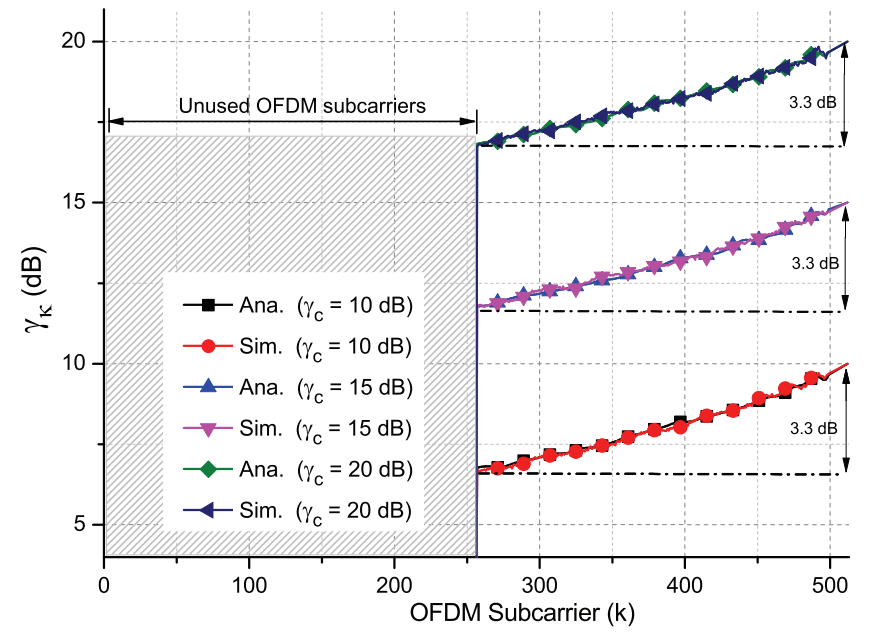

Fig. 5. Analytical and simulated OFDM subcarrier dependent SINRs $\left(\gamma_{k}\right)$ at $\gamma_{c}=10,15$, and 20dBs. Analytical $\gamma_{k}$ is given in (17), and the values are average for 10000 channel realizations.

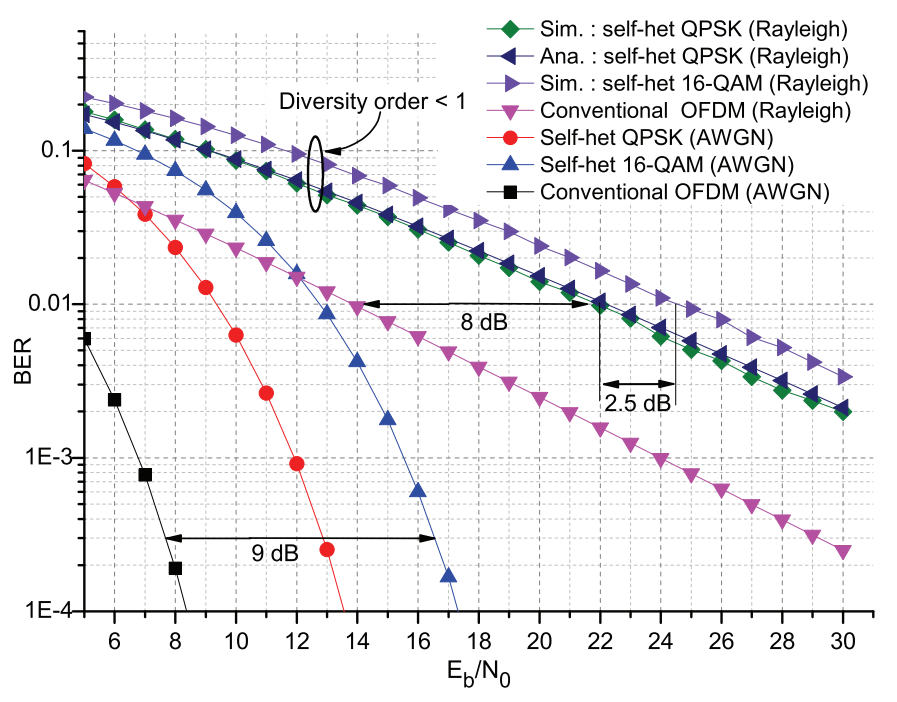

Fig. 6. BER performance comparison of the standard self-het OFDM and conventional OFDMs over AWGN and Rayleigh fading channels without phase noise.

OFDM (QPSK) with super-heterodyne structures at BER of $10^{-2}$, respectively.

In Fig. 6, for Rayleigh fading channels, we also compare the analytical BER for self-het OFDM in (18) and that by simulation using QPSK signalling. We can see that they agree with each other. Finally, we observe that the diversity order of standard self-het OFDM scheme using any constellation is less than 1 .

Fig. 7 shows the BER performance improvements given by smart carrier positioning technique for frequency selective channels. Fig. 7 also illustrates the analytical BER performance in (22) for $P=3$ and $5^{2}$. Using QPSK signalling, we observe that, both analytically and by simulation, the enhanced self-het OFDM improves the BER performance by approximately $4 \mathrm{~dB}$ at BER of $10^{-2}$ with a diversity order to

\footnotetext{
${ }^{2}$ Note that the analytical BER expression obtained for general $P$ in (22) becomes equivalent for (18) when $P=1$. Both analytical and simulation results are compared in Fig. 6 for $P=1$.
} 


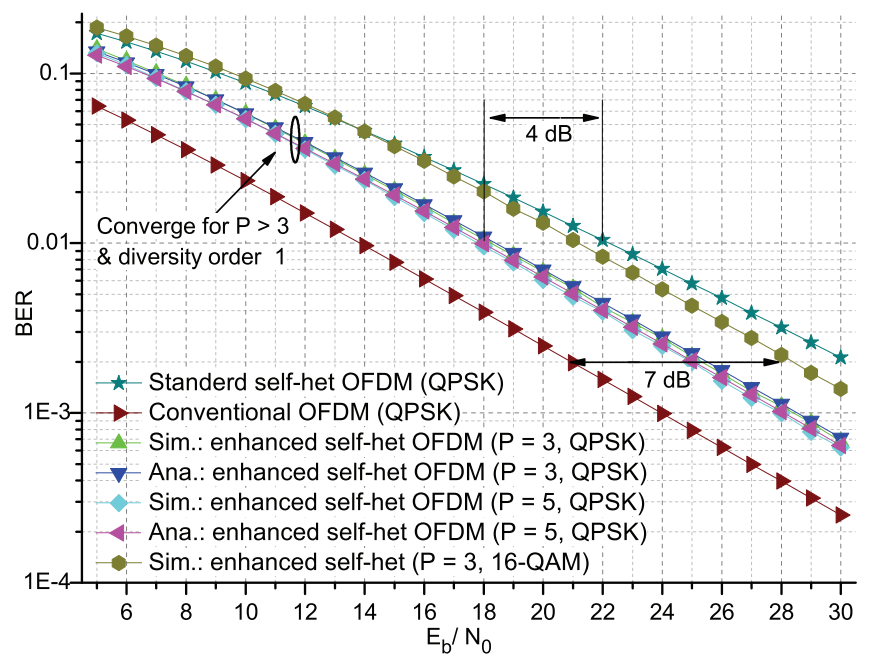

Fig. 7. BER performance comparison of the standard self-het OFDM, enhanced self-het OFDM and conventional OFDMs without phase noise.

be 1 , when compared to the standard self-het OFDM. We also observe that the BER improvement from $P=3$ to $P=5$ is very limited, and it saturates for $P>5$. As a consequence, there is no significant advantage of allocating more frequency band for the carrier. Hence, $P=3$ is a good compromise. As a result, to achieve the optimum performance, the number of potential carrier positions required by smart carrier positioning technique is very small (i.e. $P=3$ ). Hence, the rate loss due to the allocation of the potential carrier position is almost negligible for OFDM systems with large number of subcarriers (e.g. $\mathrm{N}=512$, rate loss $=3 / 512=0.58 \%$ ). Using $16-\mathrm{QAM}$ and $P=3$, we compare the BER performance of the enhanced self-het OFDM and conventional OFDM (QPSK) with superheterodyne structure under the same spectral efficiency. We observe that the SNR gap is around $7 \mathrm{~dB}$ for enhanced self OFDM scheme with the proposed smart carrier positioning technique (previously this was $10.5 \mathrm{~dB}$ for a standard self-het OFDM).

Fig. 8 compares the BER performance of enhanced selfhet OFDM $(P=3)$ and conventional OFDM scheme (with superheterodyne transceiver structures) in the presence of phase noise. To simulate the phase noise at the receiver, we adopt the OFDM phase noise model proposed in [26], where the level of oscillator instabilities is measured by the phasenoise linewidth $\beta$. We ignore the phase noise generated at the transmitter, since it is comparatively insignificant compared to the receiver phase noise and common for both OFDMs. The simulation results are presented for $N \beta / R=0,10^{-3}$ and $10^{-2}$, where $R$ is the sampling rate. As shown in Fig. 8, in the presence of phase noise, the BER performance of conventional OFDM schemes reaches an error floor at high SNRs depending upon the level of oscillator instabilities at the receiver; while enhanced self-het OFDM does not suffer from the BER error floor. Hence, self-het OFDM scheme outperforms conventional OFDM schemes with superheterodyne structures at high SNRs.

Fig. 9 shows the variation of analytical BERs in (22) with $\gamma_{c}=10 \mathrm{~dB}$ and $\eta$ for $P=1,3$, and 5 , respectively. It is shown that there exists an optimum $\eta$ (i.e. $\eta_{\mathrm{opt}}$ ) that minimizes the

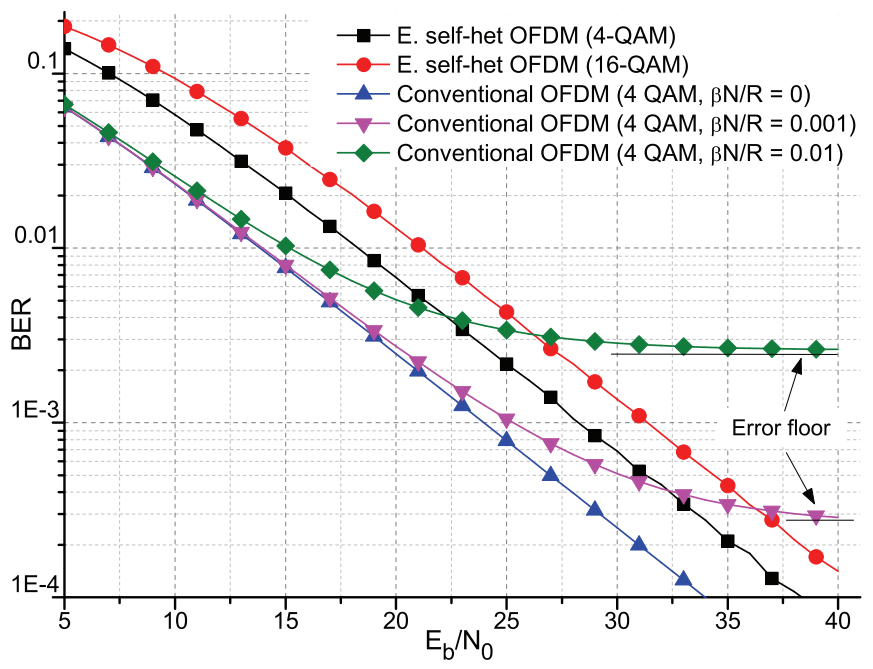

Fig. 8. BER performance comparison of the enhanced self-het OFDM and conventional OFDM (with super-heterodyne structures) in the presence of phase noise

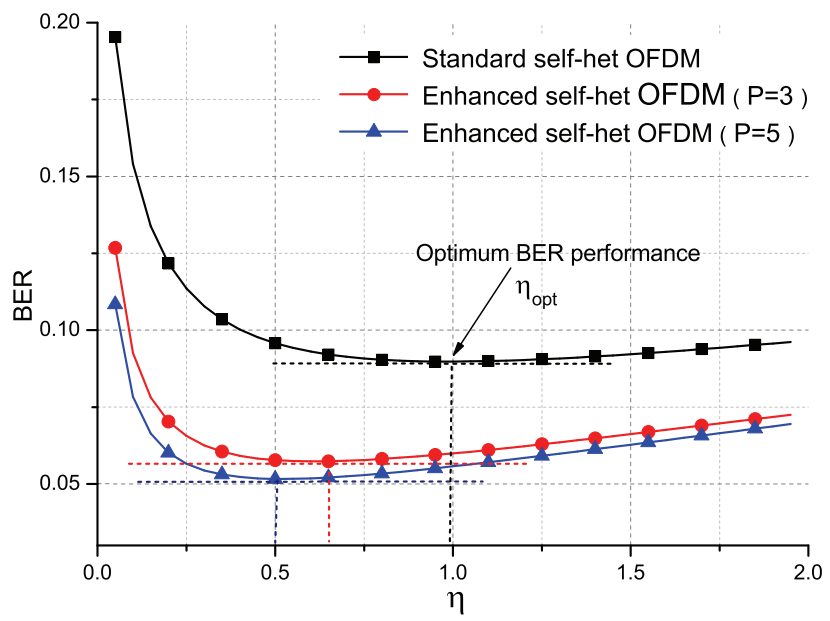

Fig. 9. BER variation with $\eta$, defined in (14), for $\gamma_{c}=10 \mathrm{~dB}$.

BER. This $\eta_{\text {opt }}$ can be obtained by solving (27) numerically. We also note that, in Fig. 9, when $\eta \geq 1$, the BER performance using both standard and enhanced self-het OFDM do not vary much, when compared to $\eta<1$. Fig. 10 shows that $\eta_{\text {opt }}$ varies with $\gamma_{c}$ using the parameters in Table I. Using the smart carrier positioning, $\eta_{\text {opt }}$ does not vary much as $\gamma_{c}$ changes.

\section{Vi. CONCLUSIONS}

In this paper, we consider and analyze the performance of self-het OFDM over frequency selective channels. We find that the standard self-het OFDM undergoes an outage if the local carrier signal experiences deep fading. This results in a large performance loss (diversity order less than one), when compared to conventional OFDM with super-heterodyne structures. We then propose the smart carrier positioning technique to mitigate this outage. It is shown that the proposed technique can improve the performance of self-het OFDM by $4 \mathrm{~dB}$ at the BER of $10^{-2}$ and restores the diversity order to be one. We showed that self-het OFDM outperforms the conventional OFDM schemes with superheterodyne receivers 


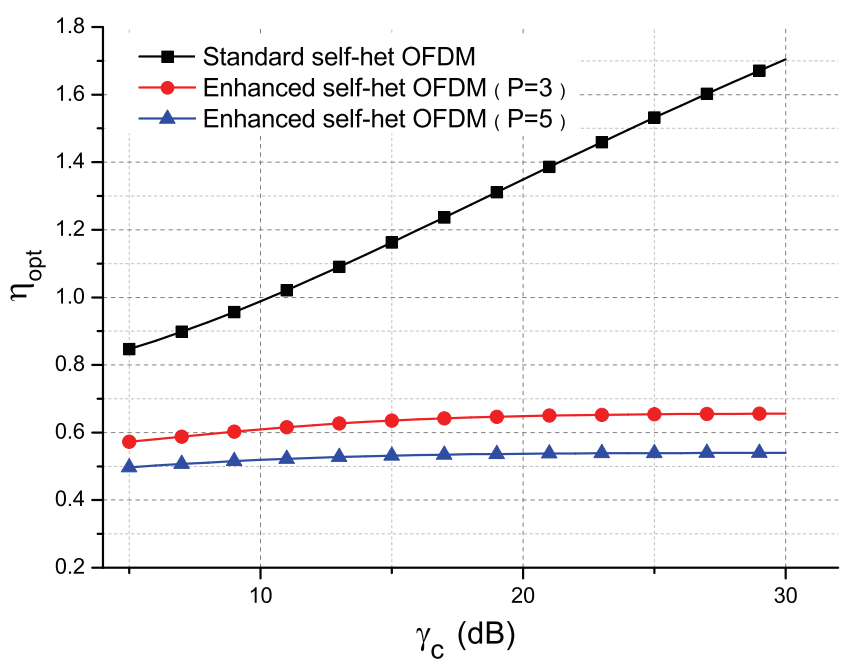

Fig. 10. The optimum power ratio between the local carrier and the OFDM subcarriers, $\eta_{\text {opt }}$ vs $\gamma_{c}$, when $P=1,3$, and 5 .

at high SNRs and does not suffer from an error floor due to phase noise. Finally, we showed that the equal power allocation between the local carrier and the OFDM subcarriers can deliver the near optimal BER performance for Rayleigh fading channels.

In millimeter/terahertz RF bands, the implementation of $\mathrm{RF}$ frontend components required for conventional OFDM (with super-heterodyne structures) is extremely challenging and expensive. In addition, we showed that conventional OFDM scheme, even with complex RF front ends, may not be effective if the level of oscillator instabilities are not sufficiently controlled. On the other hand, self-het OFDM has a simple and stable receiver that does not require a local oscillator and advanced phase locked loop circuitries. Although self-het OFDM is spectrally inefficient, it provides a good trade off between performance and complexity, when compared to the conventional OFDM with super-heterodyne structures. Hence, self-het OFDM is a promising solution to low complexity mobile communication systems using large unlicensed RF spectrum in millimeter/terahertz communication. We also demonstrate the performance of enhanced selfhet OFDM over $60 \mathrm{GHz}$ indoor RF channels in [27].

\section{APPENDIX A}

\section{ANALYTICAL BER OF SELF-HET OFDM IN RAYLEIGH FADING CHANNELS}

In this Section, we prove analytical BER expressions for both standard and enhanced self-het OFDM over Rayleigh fading channels. For large IFFT/FFT sizes, we can assume that the equivalent noise in (11) is independent of the information symbol $X_{k}$. Hence, if $M$-QAM signaling is used for the communication, the symbol error rate of $k$-th OFDM subcarrier is given by

$$
P_{k}\left(e \mid \zeta_{k}, \xi\right)=2\left(1-\frac{1}{\sqrt{M}}\right) Q\left(\sqrt{\frac{3 \gamma_{k}\left(\zeta_{k}, \xi\right)}{M-1}}\right)
$$

where $\gamma_{k}\left(\zeta_{k}, \xi\right)$ is the instantaneous SINR of the $k$-th OFDM subcarrier, given in (17). We note that $P_{k}\left(e \mid \zeta_{k}, \xi\right)$ depends on the equivalent channel gains at $k$-th OFDM subcarrier and the local carrier (i.e. $\zeta_{k}$ and $\xi$ in (15)). If the channel is Rayleigh fading, $\zeta_{k}$ follows an exponential distribution. Hence, $P_{k}\left(e \mid \zeta_{k}, \xi\right)$ averaging over $\zeta_{k}$ yields

$$
\begin{aligned}
P_{k}(e \mid \xi)= & \int_{0}^{\infty} P_{k}\left(e \mid \zeta_{k}, \xi\right) f_{\zeta_{k}}\left(\zeta_{k}\right) d \zeta_{k} \\
= & 2\left(1-\frac{1}{\sqrt{M}}\right) \int_{0}^{\infty} Q\left(\sqrt{\frac{3 \gamma_{k}\left(\zeta_{k}, \xi\right)}{M-1}}\right) e^{-\zeta_{k}} d \zeta_{k} \\
= & \left(1-\frac{1}{\sqrt{M}}\right) \times \\
& \left(1-\sqrt{\frac{3 \xi \bar{\gamma}}{2(M-1)\left(\xi+\frac{\lambda(k)}{\eta N_{x}}\right)+3 \xi \bar{\gamma}}}\right)
\end{aligned}
$$

In standard self-het OFDM, $\xi$ follows an exponential distribution. Hence, the average symbol error rate of the $k$-th OFDM subcarrier, denoted by $P_{k}(e)$, can be obtained by averaging $P_{k}(e \mid \xi)$ over $\xi$ as

$$
\begin{aligned}
P_{k}(e)= & \int_{0}^{\infty} P_{k}\left(e \mid \zeta_{k}, \xi\right) e^{-\xi} d \xi \\
= & \left(1-\frac{1}{\sqrt{M}}\right)\left(1-\frac{1}{2} \sqrt{\frac{3 \pi \bar{\gamma}}{3 \bar{\gamma}+2(M-1)}}\right. \\
& \left.\times U\left[\frac{1}{2}, 0, \frac{2(M-1) \lambda(k)}{\eta N_{x}\{3 \bar{\gamma}+2(M-1)\}}\right]\right)
\end{aligned}
$$

where $U(a, b, z)$ is the confluent hypergeometric function of the second kind and has an integral representation given by [28]

$$
U(a, b, z)=\frac{1}{\Gamma(a)} \int_{0}^{\infty} \exp (-z t) t^{a-1}(1+t)^{b-a-1} d t
$$

where $\Gamma(\cdot)$ is the gamma function. For $\bar{\gamma} \gg 1$ and Gray-coded assignment, the average bit error rate of $k$-th OFDM subcarrier can be approximate as

$$
\begin{aligned}
\mathrm{BER}_{k} \approx & 2 P_{k}(e) / \log _{2}(M) \\
\approx & \frac{2}{\log _{2}(M)}\left(1-\frac{1}{\sqrt{M}}\right)\left(1-\frac{1}{2} \sqrt{\frac{3 \pi \bar{\gamma}}{3 \bar{\gamma}+2(M-1)}}\right. \\
& \left.\times U\left[\frac{1}{2}, 0, \frac{2(M-1) \lambda(k)}{\eta N_{x}\{3 \bar{\gamma}+2(M-1)\}}\right]\right) .
\end{aligned}
$$

For an example, if QPSK signalling is used (i.e. $M=4$ ), (32) is simplified as

$$
\mathrm{BER}_{k}=\frac{1}{2}-\frac{1}{4} \sqrt{\frac{\pi \bar{\gamma}}{(2+\bar{\gamma})}} U\left(\frac{1}{2}, 0, \frac{2 \lambda(k)}{\eta N_{x}(2+\bar{\gamma})}\right)
$$

Now we consider the smart carrier positioning technique over Rayleigh fading channels. Since the equivalent channel gain in $k$-th OFDM subcarrier (i.e. $\zeta_{k}$ ) is exponentially distributed, the expressions of $P_{k}(e \mid \xi)$ in (29) is still valid. However, in smart carrier positioning technique, the distribution of $\xi$ is no longer exponential, and its pdf is given in (20) as

$$
f_{\xi}(\xi)=P e^{-\xi}\left(1-e^{-\xi}\right)^{P-1}
$$

where $P$ is total number of potential carrier positions. Hence, the new average symbol error rate of the $k$-th OFDM subcarrier, denoted by $P_{k}^{S}(e)$, can be obtaining by averaging $P_{k}(e \mid \xi)$ 
over $\xi$ as

$$
\begin{aligned}
P_{k}^{S}(e)= & \int_{0}^{\infty} P_{k}(e \mid \xi) f_{\xi}(\xi) d \xi \\
= & \left(1-\frac{1}{\sqrt{M}}\right)\left(1-\frac{1}{2} \sqrt{\frac{3 \pi \bar{\gamma}}{3 \bar{\gamma}+2(M-1)}} \sum_{t=1}^{P}\left(\begin{array}{c}
P \\
t
\end{array}\right)\right. \\
& \left.\times(-1)^{t-1} U\left[\frac{1}{2}, 0, \frac{2(M-1) \lambda(k) t}{\eta N_{x}\{3 \bar{\gamma}+2(M-1)\}}\right]\right) .
\end{aligned}
$$

As in (32), the approximated bit error rate of $k$-th OFDM subcarrier can be estimated by

$$
\begin{gathered}
\operatorname{BER}_{k}^{S} \approx \frac{2}{\log _{2}(M)}\left(1-\frac{1}{\sqrt{M}}\right)\left(1-\frac{1}{2} \sqrt{\frac{3 \pi \bar{\gamma}}{3 \bar{\gamma}+2(M-1)}}\right. \\
\left.\sum_{t=1}^{P}\left(\begin{array}{c}
P \\
t
\end{array}\right)(-1)^{t-1} U\left[\frac{1}{2}, 0, \frac{2(M-1) \lambda(k) t}{\eta N_{x}\{3 \bar{\gamma}+2(M-1)\}}\right]\right) \cdot(35)
\end{gathered}
$$

If QPSK signalling is used, (35) is simplified as

$$
\begin{aligned}
\operatorname{BER}_{k}^{S}= & \frac{1}{2}-\frac{1}{4} \sqrt{\frac{\pi \bar{\gamma}}{2+\bar{\gamma}}} \times \\
& \sum_{t=1}^{P}\left(\begin{array}{l}
P \\
t
\end{array}\right)(-1)^{t-1} U\left(\frac{1}{2}, 0, \frac{2 t \lambda(k)}{\eta N_{x}(2+\bar{\gamma})}\right) .
\end{aligned}
$$

Similar analysis can be done for Nakagami Fading channels [29].

\section{ACKNOWLEDGMENT}

This work was performed at the Monash Software Defined Telecommunications Lab and supported by the Monash Professional Fellowship and Australian Research Councils Discovery grant under DP 130100336.

\section{REFERENCES}

[1] A. Armada and M. Calvo, "Phase noise and sub-carrier spacing effects on the performance of an OFDM communication system," IEEE Commun. Lett., vol. 2, no. 1, pp. 11-13, Jan. 1998.

[2] Z. Lao, J. Jensen, K. Guinn, and M. Sokolich, "80-GHz differential VCO in InP SHBTs," IEEE Microwave and Wireless Components Lett., vol. 14, no. 9, pp. 407-409, Sept. 2004.

[3] C. Cao and K. O, "Millimeter-wave voltage-controlled oscillators in 0.13 $\mu m$ CMOS technology," IEEE J. Solid-State Circuits, vol. 41, no. 6, pp. 1297-1304, June 2006

[4] N. Fernando, Y. Hong, and E. Viterbo, "Flip-OFDM for optical wireless communications," in Proc. 2011 IEEE Information Theory Workshop, pp. 5-9.

[5] N. Fernando, Y. Hong, and E. Viterbo, "Flip-OFDM for unipolar communication systems," IEEE Trans. Commun., vol. 60, no. 12, pp. 3726-3733, Dec. 2012.

[6] Y. Shoji, M. Nagatsuka, K. Hamaguchi, and H. Ogawa, "60 GHz band 64 QAM/OFDM terrestrial digital broadcasting signal transmission by using millimeter-wave self-heterodyne system," IEEE Trans. Broadcast., vol. 47, no. 3, pp. 218-227, Sept. 2001.

[7] Y. Shoji, K. Hamaguchi, and H. Ogawa, "Millimeter-wave remote selfheterodyne system for extremely stable and low-cost broad-band signal transmission," IEEE Trans. Microwave Theory and Techniques, vol. 50, no. 6, pp. 1458-1468, June 2002.

[8] R. Pacheco and D. Hatzinakos, "BER analysis of self-heterodyne OFDM transmission scheme," in Proc. 2004 Canadian Conference on Electrical and Computer Engineering, vol. 4, pp. 1953-1956.

[9] Y. Shoji, C. Choi, and H. Ogawa, "70-GHz-Band OFDM transceivers based on Self-Heterodyne scheme for millimeter-wave wireless personal area network," IEEE Trans. Microwave Theory and Techniques, vol. 57, no. 10 , pp. 2940-2948, Oct. 2009.

[10] A. Lowery and J. Armstrong, "Orthogonal-frequency-division multiplexing for dispersion compensation of long-haul optical systems," Opt. Express, vol. 14, no. 6, pp. 2079-2084, Mar. 2006.
[11] A. J. Lowery, "Amplified-spontaneous noise limit of optical OFDM lightwave systems," Opt. Express, vol. 16, no. 2, pp. 860-865, Jan. 2008.

[12] Y. Shoji and H. Ogawa, "High-receiving-sensitivity 70-GHz band MMIC transceiver: application of receiving-module-arrayed self-heterodyne technique," in 2004 IEEE MTT-S International Microwave Symposium Digest, vol. 1, pp. 219-222.

[13] C. Choi, Y. Shoji, and H. Ogawa, "Analysis of receiver space diversity gain for millimeter-wave self-heterodyne transmission techniques under two-path channel environments," in Proc. 2007 IEEE Radio and Wireless Symposium, pp. 75-78.

[14] C. Choi and Y. Shoji, "Third-order intermodulation distortion characteristics of millimeter-wave self-heterodyne transmission techniques," in Proc. 2006 Asia-Pacific Microwave Conference, pp. 1751-1756.

[15] Y. Shoji, C. Choi, and H. Ogawa, "Millimeter-wave OFDM WPAN system applying adaptive modulation for grouped sub-carriers," in Proc. 2007 IEEE Radio and Wireless Symposium, pp. 499-502.

[16] C. Choi, Y. Shoji, and H. Ogawa, "Implementation of an OFDM baseband with adaptive modulations to grouped subcarriers for millimeterwave wireless indoor networks," IEEE Trans. Consum. Electron., vol. 57, no. 4, pp. 1541-1549, Nov. 2011.

[17] H. Song and T. Nagatsuma, "Present and future of terahertz communications," IEEE Trans. Terahertz Science and Technol., vol. 1, no. 1, pp. 256-263, Sept. 2011.

[18] Y. Haibing, P. F. M. Smulders, and M. H. A. J. Herben, "Frequency selectivity of $60-\mathrm{GHz}$ LOS and NLOS indoor radio channels," in Proc. 2006 IEEE Vehicular Technology Conference - Spring, pp. 2727-2731.

[19] P. Smulders, "Statistical Characterization of $60-\mathrm{GHz}$ Indoor Radio Channels," IEEE Trans. Antennas Propag., vol. 57, no. 10, pp. 2820 2829, Oct. 2009

[20] C. Liu, E. Skafidas, T. S. Pollock, and R. J. Evans, "Angle of arrival extended S-V model for the $60 \mathrm{Ghz}$ wireless desktop channel," in Proc. 2006 IEEE Personal, Indoor and Mobile Radio Communications, pp. 1114.

[21] Y. Shoji, H. Sawada, C. Chang-Soon, and H. Ogawa, "A modified SVmodel suitable for line-of-sight desktop usage of millimeter-wave WPAN systems," IEEE Trans. Antennas Propag., vol. 54, no. 10, pp. 3664-3674, Oct. 2006.

[22] J. P. Hoon, K. Yungsoo, H. S. Young, L. Kyutae, and K. Ki-Ho, "Analysis of $60 \mathrm{GHz}$ band indoor wireless channels with channel configurations," in Proc. 1998 IEEE International Symposium on Personal, Indoor and Mobile Radio Communications, vol. 2, pp. 617-620.

[23] N. Moraitis and P. Constantinou, "Measurements and characterization of wideband indoor radio channel at $60 \mathrm{GHz}$," IEEE Trans. Wireless Commun., vol. 5, no. 4, pp. 880-889, Apr. 2006

[24] Y. Hong, E. Viterbo, and A. J. Lowery, "Improving the sensitivity of direct-detection optical OFDM systems by pairing of the optical subcarriers," in Proc. 2011 European Conference and Exposition on Optical Communications, pp. 1-3.

[25] Y. Hong, A. J. Lowery, and E. Viterbo, "Sensitivity improvement and carrier power reduction in direct-detection optical OFDM systems by subcarrier pairing," Opt. Express, vol. 20, no. 2, pp. 1635-1648, Jan. 2012.

[26] S. Wu and Y. Bar-Ness, "OFDM systems in the presence of phase noise: consequences and solutions," IEEE Trans. Commun., vol. 52, no. 11, pp. 1988-1996, Nov. 2004.

[27] N. Fernando, Y. Hong, and E. Viterbo, "Analysis of self-het OFDM enhancements for $60 \mathrm{GHz}$ indoor RF channels," in 2013 Australian Communications Theory Workshop.

[28] G. Kristensson, "Second Order Differential Equations: Special Functions and Their Classification," 1st edition. Springer, 2010.

[29] K. A. Hamdi, "A useful technique for interference analysis in Nakagami fading," IEEE Trans. Commun., vol. 55, no. 6, pp. 1120-1124, Jun. 2007.

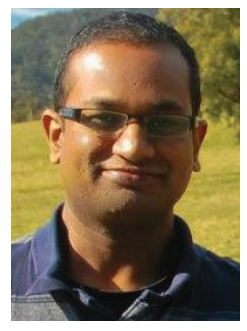

Nirmal Fernando received the B.Sc (Hones), and PG. Dip degrees from the Department of Electronic and Telecommunication Engineering, University of Moratuwa, Sri Lanka, in 2009 and 2009, respectively. He is currently working towards the Ph.D. degree at the Department of Electrical and Computer Systems Eng., at Monash University, Melbourne, Australia. From 2009 to 2010, he has been working as a lecturer at University of Moratuwa, Sri Lanka, from 2006 to 2009, as an Engineer in Dialog Telekom, Sri Lanka. His research interests include non-coherent OFDM techniques, low complexity RF receivers, Optical OFDM, MIMO precoding, signal processing in wireless communications and communication theory. 


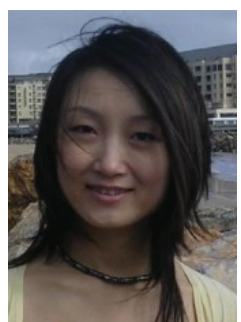

Yi Hong is currently a lecturer at the Department of Elecectrical and Computer Systems Eng., at Monash University, Melbourne, Australia. She received her Ph.D. degree in Electrical Engineering and Telecommunications from the University of New South Wales (UNSW), Sydney, Australia, in Oct. 2004. She then worked at the Institute of Telecom. Research, University of South Australia, Australia; at the Institute of Advanced Telecom., Swansea University, UK; and at University of Calabria, Italy.

During her Ph.D., she received an International Postgraduate Research Scholarship (IPRS) from the Commonwealth of Australia; a supplementary Engineering Award from the School of Electrical Engineering and Telecommunications, UNSW; and a Wireless Data Communication System Scholarship from UNSW. She received the NICTA-ACoRN Earlier Career Researcher award for a paper presented at the Australian Communication Theory Workshop (AUSCTW), Adelaide, Australia, 2007.

Dr. Hong is an Associate Editor for European Transactions on Telecommunications and an IEEE Senior member. She was Technical Program Committee Chair of AUSCTW'11, Melbourne, Australia. She was the Publicity Chair at the IEEE Information Theory Workshop 2009, Sicily, Italy. She is a Technical Program Committee member for various IEEE conferences such as IEEE ICC 2011, VTC 2011, PIMRC and WCNC 2008. Her research interests include information and communication theory with applications to telecommunication engineering.

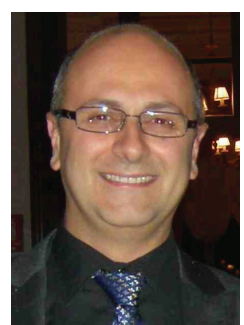

Emanuele Viterbo was born in Torino, Italy, in 1966. He received his degree (Laurea) in Electrical Engineering in 1989 and his Ph.D. in 1995 in Electrical Engineering, both from the Politecnico di Torino, Torino, Italy. From 1990 to 1992 he was with the European Patent Office, The Hague, The Netherlands, as a patent examiner in the field of dynamic recording and error-control coding. Between 1995 and 1997 he held a post-doctoral position in the Dipartimento di Elettronica of the Politecnico di Torino. In 1997-98 he was a post-doctoral research fellow in the Information Sciences Research Center of AT\&T Research, Florham Park, NJ, USA. He became first Assistant Professor (1998) then Associate Professor (2005) in Dipartimento di Elettronica at Politecnico di Torino. In 2006 he became Full Professor in DEIS at University of Calabria, Italy. Since September 2010 he is Full Professor in the ECSE Department and Associate Dean in Research Training at Monash University, Melbourne, Australia.

Prof. Emanuele Viterbo is an ISI Highly Cited Researcher since 2009. He is Associate Editor of IEEE TRANSACTIONS ON INFORMATION THEORY, European Transactions on Telecommunications and Journal of Communications and Networks, and Guest Editor for IEEE JOURNAL OF SELECTED TOPICS In Signal Processing Special Issue on Managing Complexity in Multiuser MIMO Systems.

In 1993 he was visiting researcher in the Communications Department of DLR, Oberpfaffenhofen, Germany. In 1994 and 1995 he was visiting the Ecole Nationale Supérieure des Télécommunications (E.N.S.T.), Paris. In 2003 he was visiting researcher at the Maths Department of EPFL, Lausanne, Switzerland. In 2004 he was visiting researcher at the Telecommunications Department of UNICAMP, Campinas, Brazil. In 2005, 2006 and 2009 he was visiting researcher at the ITR of UniSA, Adelaide, Australia. In 2007 he was visiting fellow at the Nokia Research Center, Helsinki, Finland.

Dr Emanuele Viterbo was awarded a NATO Advanced Fellowship in 1997 from the Italian National Research Council. His main research interests are in lattice codes for the Gaussian and fading channels, algebraic coding theory, algebraic space-time coding, digital terrestrial television broadcasting, digital magnetic recording, and irregular sampling. 